\title{
Annexin A5 inhibits diffuse large B-cell lymphoma cell invasion and chemoresistance through phosphatidylinositol 3-kinase signaling
}

\author{
JINGJING WANG $^{1}$, YANG ZHANG ${ }^{1}$, XIANLING LIU ${ }^{1}$, JINAN MA ${ }^{1}$, \\ PING LIU ${ }^{1}$, CHUNHONG HU ${ }^{1}$ and GUANGSEN ZHANG ${ }^{2}$ \\ Departments of ${ }^{1}$ Oncology and ${ }^{2}$ Hematology, The Second Xiangya Hospital, \\ Central South University, Changsha, Hunan 410011, P.R. China
}

Received March 5, 2014; Accepted May 20, 2014

DOI: $10.3892 /$ or.2014.3547

\begin{abstract}
Diffuse large B-cell lymphoma (DLBCL) is the most common type of non-Hodgkin's lymphoma worldwide. Although patient outcomes have significantly improved to a greater than $40 \%$ cure rate by the combinatorial cyclophosphamide, doxorubicin, vincristine and prednisone (CHOP) chemotherapy, which is widely used, resistance to the CHOP regimen continues to pose a problem in managing or curing DLBCL. While it promotes the malignancy and chemoresistance in certain types of cancer, Annexin A5 is negatively correlated with those in other cancers, including DLBCL. In the present study, we explored the effects of Annexin A5 on DLBCL cell invasion and chemoresistance to CHOP. Stable overexpression and knockdown of Annexin A5 were performed in Toledo and Pfeiffer human DLBCL cell lines. Overexpression of Annexin A5 in both cell lines significantly decreased cell invasion, matrix metalloproteinase-9 (MMP-9) expression/activity, phosphatidylinositol 3-kinase (PI3K) activity/Akt phosphorylation, and cell survival against CHOP-induced apoptosis. On the other hand, knockdown of Annexin A5 markedly increased cell invasion, MMP-9 expression/activity, PI3K activity/Akt phosphorylation, and CHOP-induced apoptosis in the DLBCL cell lines, which was abolished by selective PI3K inhibitor BKM120. In conclusion, our study provides the first in vitro evidence that Annexin A5 inhibits DLBCL cell invasion, MMP-9 expression/activity, and chemoresistance to $\mathrm{CHOP}$ through a PI3K-dependent
\end{abstract}

Correspondence to: Dr Chunhong Hu, Department of Oncology, The Second Xiangya Hospital, Central South University, 139 Middle Renmin Road, Changsha, Hunan 410011, P.R. China

E-mail: huchunh@medmail.com.cn

Dr Guangsen Zhang, Department of Hematology, The Second Xiangya Hospital,Central South University, 139 Middle Renmin Road, Changsha, Hunan 410011, P.R. China

E-mail: zgsllzy@163.com

Key words: diffuse large B-cell lymphoma, Annexin A5, invasion, chemoresistance, CHOP chemotherapy, phosphatidylinositol3-kinase mechanism; it provides new insight not only into the biological function of Annexin A5, but also into the molecular mechanisms underlying DLBCL progression and chemoresistance.

\section{Introduction}

Diffuse large B-cell lymphoma (DLBCL) is the most common type of non-Hodgkin's lymphoma (NHL) worldwide (1). DLBCL represents a heterogeneous group of tumors with a high variance of genetic abnormalities, clinical features, response to treatment and prognosis (2). Combinatorial cyclophosphamide, doxorubicin, vincristine and prednisone (CHOP) chemotherapy has been a systemic therapy for DLBCL with a cure rate of $40-50 \%$ (3) and is widely used in China. Although a subset of DLBCL patients is cured with CHOP regimens, many succumb to chemorefractory disease (4). Resistance to the CHOP anthracycline-based regimen continues to be a serious challenge in the cure of DLBCL (3). The molecular basis for development of the multi-drug chemoresistance in DLBCL remains unclear.

Annexins are predominantly cytosolic soluble proteins that can reversibly bind to negatively charged phospholipids in a $\mathrm{Ca}^{2+}$-mediated manner. Twelve Annexins common to vertebrates are known as Annexins A1-A11 and A13 $(5,6)$. Annexin A5, also known as placental anticoagulant protein I, thromboplastin inhibitor V, endonexin II, calphobindin I and lipocortin V, was first described functionally as a vascular anticoagulant in 1985 (7,8). Annexin A5 deregulations were observed as causative phenomena in a range of physiological and pathological processes. Annexin A5 reportedly promotes tumorigenesis and progression of a variety of cancers, including hepatocarcinoma, breast, colorectal, pancreatic, bladder and prostate cancer (9). Nevertheless, upregulation of Annexin A5 has also been found to be negatively correlated with the malignancy of thyroid cancer (9). Previous studies suggested that Annexin A5 enhances chemoresistance in gastric cancer and nasopharyngeal carcinoma $(10,11)$. However, a recent study showed that Annexin A5 was upregulated in CHOP-sensitive DLBCL tissues, suggesting that Annexin A5 may inhibit chemoresistance in DLBCL (12). 
Our pilot studies suggested that Annexin A5 could increase chemosensitivity in DLBCL cells. In the present study, we explored the effects of Annexin A5 on DLBCL cell invasion and chemoresistance to CHOP.

\section{Materials and methods}

Cells lines and reagents. Toledo (CRL-2631) and Pfeiffer (CRL-2632) human DLBCL cell lines were purchased from the American Type Culture Collection (Manassas, VA, USA). Annexin A5 shRNA lentiviral particles (sc-29686-V), control shRNA lentiviral particles-A (sc-108080), selective phosphatidylinositol 3-kinase (PI3K) inhibitor BKM120 (sc-364437A), anti-Annexin A5 antibody (sc-74438), anti-matrix metalloproteinase-9 (MMP-9) antibody (sc-21733), and anti-Akt (5C10) (sc-81434) and anti-P-Akt (ser473) (sc-101629) antibodies were purchased from Santa Cruz Biotechnology (Santa Cruz, CA, USA). The SensoLyte ${ }^{\circledR} 520$ MMP-9 Assay kit (71155) was purchased from AnaSpec (Fremont, CA, USA). The QCM ECMatrix 24-well $(8 \mu \mathrm{M})$ Fluorimetric Cell Invasion assay kit (ECM554) was purchased from Chemicon (Millipore, Billerica, MA, USA). The TiterTACS in situ apoptosis detection kit (4822-96-K) was purchased from R\&D Systems (Minneapolis, MN, USA). The PI3K Activity ELISA kit (K-1000s) was purchased from Echelon Biosciences (Salt Lake City, UT, USA). Superfect ${ }^{\mathrm{TM}}$ transfection reagent was purchased from Qiagen (Valencia, CA, USA). The Annexin A5 expression vector (RC205619), in which the full-length human Annexin A5 cDNA was subcloned into the pCMV6-entry vector, was purchased from Origene (Beijing, China). Puromycin, G418, cyclophosphoramide, doxorubicin, vincristine, prednisone and all chemicals of reagent grade were purchased from Sigma (St. Louis, MO, USA). Cyclophosphoramide and doxorubicin were dissolved in Millipore-purified water, vincristine was dissolved in methanol, and prednisone was dissolved in chloroform/ethanol (1:1). All CHOP reagents were stored at $-80^{\circ} \mathrm{C}$.

Transfection and lentiviral transduction. The Annexin A5 expression vector was transfected into cells using Superfect ${ }^{\mathrm{TM}}$ transfection reagent (Qiagen) according to the manufacturer's instructions. Pools of stable transductants were generated via selection with G418 $(600 \mu \mathrm{g} / \mathrm{ml})$ following the manufacturer's protocol. The Annexin A5 shRNA lentiviral particles contain expression constructs encoding target-specific 19-25 nt (plus hairpin) shRNA designed to specifically knock down Annexin A5 gene expression. The control shRNA lentiviral particles contain a scrambled shRNA sequence that will not lead to specific degradation of any cellular mRNA, and was used as a negative control for Annexin A5 shRNA lentiviral particles. Lentiviral transduction was performed in Toledo and Pfeiffer cells. Pools of stable transductants were generated via selection with puromycin $(4 \mu \mathrm{g} / \mathrm{ml})$ according to the manufacturer's protocol (Santa Cruz Biotechnology).

Cell invasion assay. In vitro cell invasion assays were performed with the QCM ECMatrix ${ }^{\circledR} 24$-well $(8 \mu \mathrm{M})$ Fluorimetric Cell Invasion assay kit (Chemicon; Millipore) according to the manufacturer's instructions $(13,14)$. The kit used an insert polycarbonate membrane with an $8-\mu \mathrm{M}$ pore size. The insert in the invasion kit was coated with a thin layer of ECMatrix. Cell invasion was determined by fluorescence. Each experiment was repeated three times in duplicates.

Western blot analysis. Cells dissolved in $250 \mu 1$ of 2X SDS loading buffer $(62.5 \mathrm{~mm}$ TrisHCl, pH 6.8, 2\% SDS, 25\% glycerol, $0.01 \%$ bromophenol blue, 5\% 2-mercaptoethanol), and incubated at $95^{\circ} \mathrm{C}$ for $10 \mathrm{~min}$. Equal amounts of proteins for each sample were separated by $10 \%$ SDS-polyacrylamide gel and blotted onto a polyvinylidene difluoride microporous membrane (Millipore). Membranes were incubated for $1 \mathrm{~h}$ with a 1/500 dilution of anti-MMP-9, anti-P-Akt (ser473) or anti-Akt antibody, and then washed and revealed using secondary antibodies with horseradish peroxidase conjugate $(1 / 4000,1$ h). Peroxidase was revealed with a GE Healthcare ECL kit (Shanghai, China). Three independent experiments were performed for each western blot analysis.

MMP-9 activity assay. MMP-9 activity was measured with the SensoLyte 520 MMP-9 assay kit (AnaSpec) according to the manufacturer's instructions $(15,16)$. The supernatants were collected and then incubated with 4-aminophenylmercuric acetate (AMPA) and MMP-9 substrate. The fluorescence intensity at Ex/Em wave lengths of 490/520 nm were used as a measure of MMP-9 activity. Each experiment was repeated three times in duplicates.

Cell apoptosis assay. Cells were cultured at $9 \times 10^{4}$ cells/well in 96 -well tissue culture plates and incubated at $37^{\circ} \mathrm{C}$ for 24 or $48 \mathrm{~h}$ under $\mathrm{CHOP}$ treatment. The composition of $\mathrm{CHOP}$ consisted of cyclophosphoramide, doxorubicin, vincristine and prednisone at the clinical ratio of 80/5.5/0.16/11.1 (3), with the combined CHOP concentration set at $80 \mathrm{ng} / \mathrm{ml}$. Cell apoptosis was measured at 24 and $48 \mathrm{~h}$ with a microplate reader-based TiterTACS in situ apoptosis detection kit (R\&D Systems) as described by the manufacturer. Each experiment was repeated three times in triplicates.

PI3K activity assay. PI3K activity was determined with the PI3K Activity ELISA kit (Echelon Biosciences) according to the manufacturer's instructions $(17,18)$. For direct functional assessment of PI3K activity, PI3K was isolated by immunoprecipitation using an anti-PI3K antibody (Millipore, \#06-195) to the p85 adapter subunit, and the ability of the co-precipitated catalytic p110 catalytic subunit to convert a standard PIP2 to PIP3 in a kinase reaction was assessed by measuring the generated PIP3 by the ELISA kit. Each experiment was repeated three times in duplicates.

Statistical analysis. Statistical analyses were performed with SPSS for Windows 10.0. All data values are expressed as means $\pm \mathrm{SD}$. Comparisons of means among multiple groups were performed with one-way ANOVA followed by post hoc pairwise comparisons using Tukey's tests. A two-tailed $\mathrm{p}<0.05$ was considered to indicate statistically significant differences.

\section{Results}

Overexpression and knockdown of Annexin A5 in human DLBCL cells. We used Toledo and Pfeiffer human DLBCL cells as cell models in this study. The Toledo and the Pfeiffer 
A
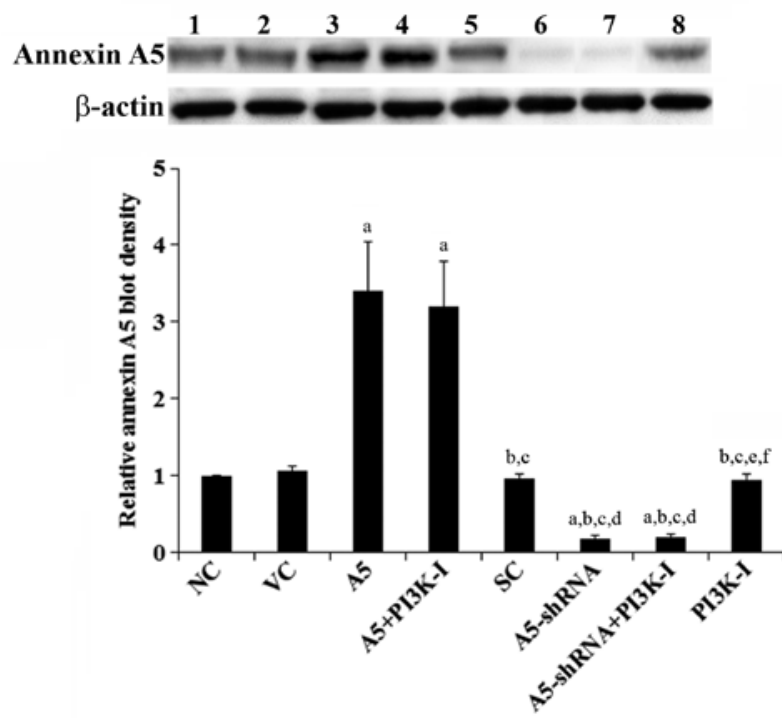

B
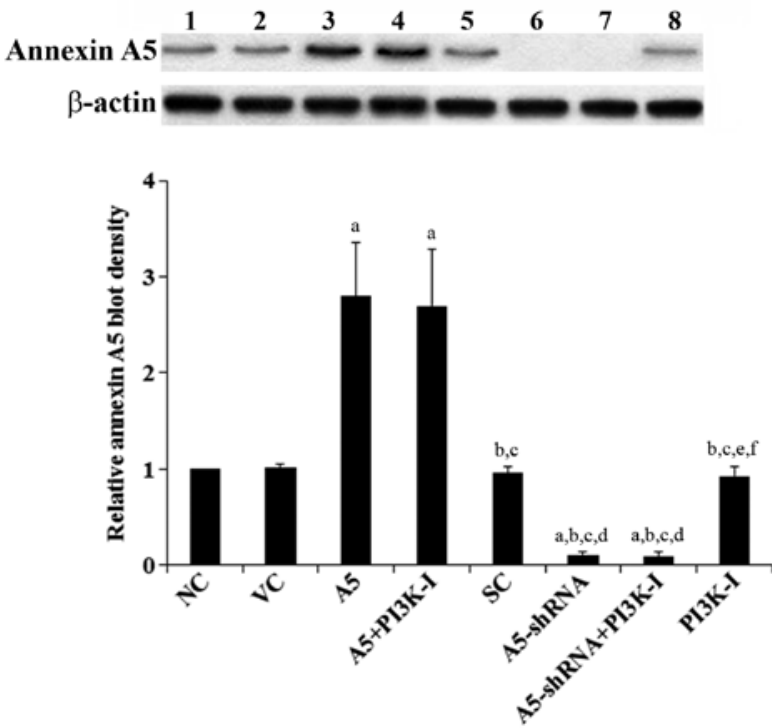

Figure 1. Annexin A5 expression in human diffuse large B-cell lymphoma (DLBCL) cells with overexpression or knockdown of Annexin A5. In (A) Toledo and (B) Pfeiffer human DLBCL cells, expression of Annexin A5 in normal control cells (NC, lane 1), cells stably transfected with the empty pCMV6-entry vector (VC, lane 2), cells stably transfected with Annexin A5 with or without phosphatidylinositol 3-kinase (PI3K) inhibitor BKM120 $(50 \mu \mathrm{M})$ treatment (A5, lane 3; A5+PI3K-I, lane 4), cells stably transduced with scramble control shRNA (SC, lane 5), cells stably transduced with Annexin A5-shRNA with or without BKM120 $(50 \mu \mathrm{M})$ treatment (A5-shRNA, lane 6; A5-shRNA+PI3K-I, lane 7), and cells treated with BKM120 $(50 \mu \mathrm{M})$ (PI3K-I, lane 8) was analyzed with western blot analysis. $\beta$-actin blotting was used as a loading control. Density of the Annexin A5 blot was normalized against that of the $\beta$-actin blot to obtain a relative Annexin A5 blot density, which was expressed as fold changes to that of NC (designated as 1). Three independent experiments were performed for each western blot analysis. Data values are expressed as mean \pm SD. ${ }^{\mathrm{a}} \mathrm{p}<0.05 \mathrm{com}-$ pared with $\mathrm{NC}$ and $\mathrm{VC} ;{ }^{b} \mathrm{p}<0.05$ compared with A5; ${ }^{\mathrm{c}} \mathrm{p}<0.05$ compared with A $5+\mathrm{PI} 3 \mathrm{~K}-\mathrm{I} ;{ }^{\mathrm{d}} \mathrm{p}<0.05$ compared with $\mathrm{SC} ;{ }^{\mathrm{e}} \mathrm{p}<0.05$ compared with A5-shRNA; ${ }^{\mathrm{f}} \mathrm{p}<0.05$ compared with A5-shRNA+PI3K-I.

cell lines were established from peripheral blood leukocytes and metastatic site (pleural effusion) of patients with DLBCL, respectively. Western blot analyses revealed that Toledo cells had higher constitutive Annexin A5 expression than Pfeiffer (Fig. 1). We overexpressed and knocked down Annexin A5 in both cell lines by stable transfection of an Annexin A5 expres-
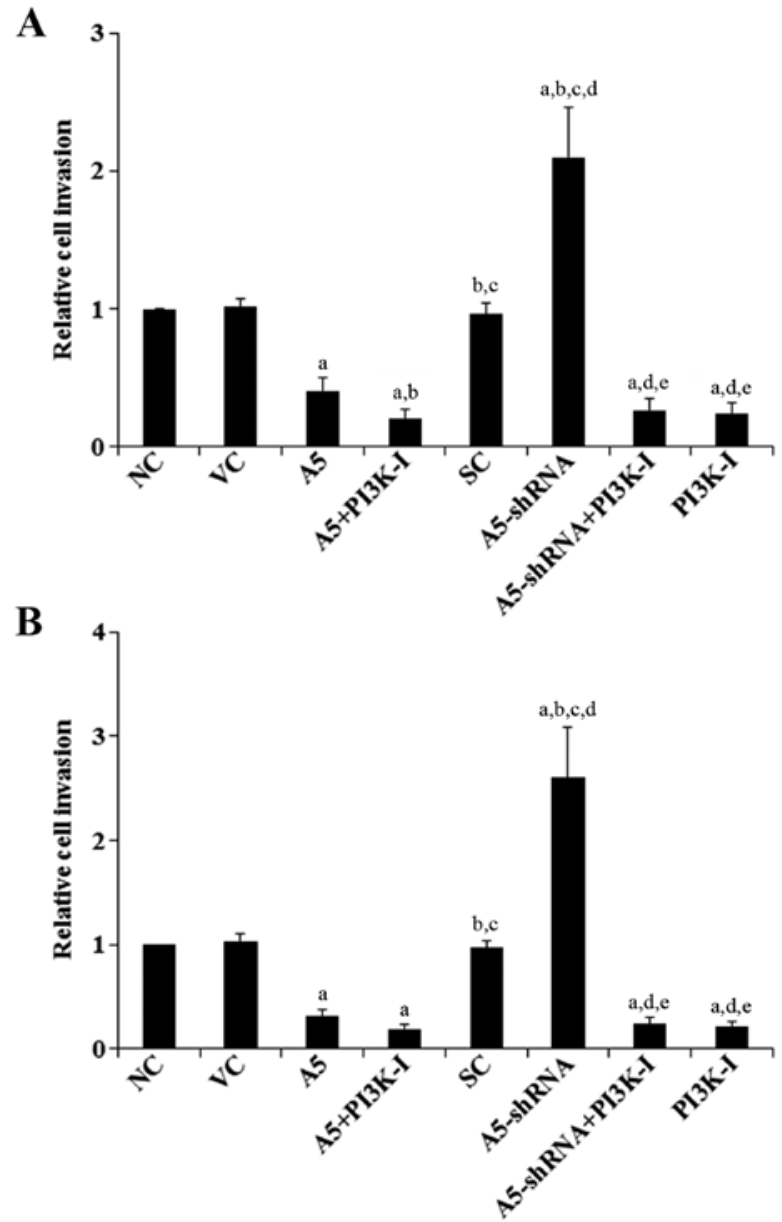

Figure 2. Effects of Annexin A5 on diffuse large B-cell lymphoma (DLBCL) cell invasion. In vitro cell invasion assays were performed with a Fluorimetric Cell Invasion Assay kit (Chemicon; Millipore) in (A) Toledo and (B) Pfeiffer DLBCL cells. Cell invasion in normal control cells (NC), cells stably transfected with the empty pCMV6-entry vector (VC), cells stably transfected with Annexin A5 with or without phosphatidylinositol 3-kinase (PI3K) inhibitor BKM120 (50 $\mu \mathrm{M})$ treatment (A5; A5+PI3K-I), cells stably transduced with scramble control shRNA (SC), cells stably transduced with Annexin A5-shRNA with or without BKM120 (50 $\mu \mathrm{M})$ treatment (A5-shRNA; A5-shRNA+PI3K-I), and cells treated with BKM120 $(50 \mu \mathrm{M})(\mathrm{PI} 3 \mathrm{~K}-\mathrm{I})$ was determined by fluorescence and shown as fold changes to that of NC (designated as 1). Each experiment was repeated three times in duplicates. Data values are expressed as mean $\pm \mathrm{SD}$. ${ }^{\mathrm{a}} \mathrm{p}<0.05$ compared with $\mathrm{NC}$ and $\mathrm{VC} ;{ }^{\mathrm{b}} \mathrm{p}<0.05$ compared with $\mathrm{A} 5 ;{ }^{\mathrm{c}} \mathrm{p}<0.05$ compared with A5+PI3K-I; ${ }^{\mathrm{d}} \mathrm{p}<0.05$ compared with $\mathrm{SC} ;{ }^{\mathrm{e}} \mathrm{p}<0.05$ compared with A5-shRNA.

sion vector and lentiviral transduction of Annexin A5-shRNA, respectively. As shown in Fig. 1, compared with the controls, Annexin A5 was overexpressed 3.4- and 2.8-fold in Toledo and Pfeiffer cells, respectively. On the other hand, the endogenous Annexin A5 level was knocked down 81 and $89 \%$ in Toledo and Pfeiffer cells, respectively (Fig. 1). As our pilot studies suggested that Annexin A5 would regulate DLBCL cell invasion and chemosensitivity through a PI3K-dependent mechanism (data not shown), we included a selective PI3K inhibitor BKM120 $(50 \mu \mathrm{M})$ in all experiments in this study. As shown in Fig. 1, the PI3K inhibitor had no significant effect on Annexin A5 expression in both Toledo and Pfeiffer cells.

Effects of Annexin A5 on DLBCL cell invasion and MMP-9 expression/activity. To examine the effect of Annexin A5 

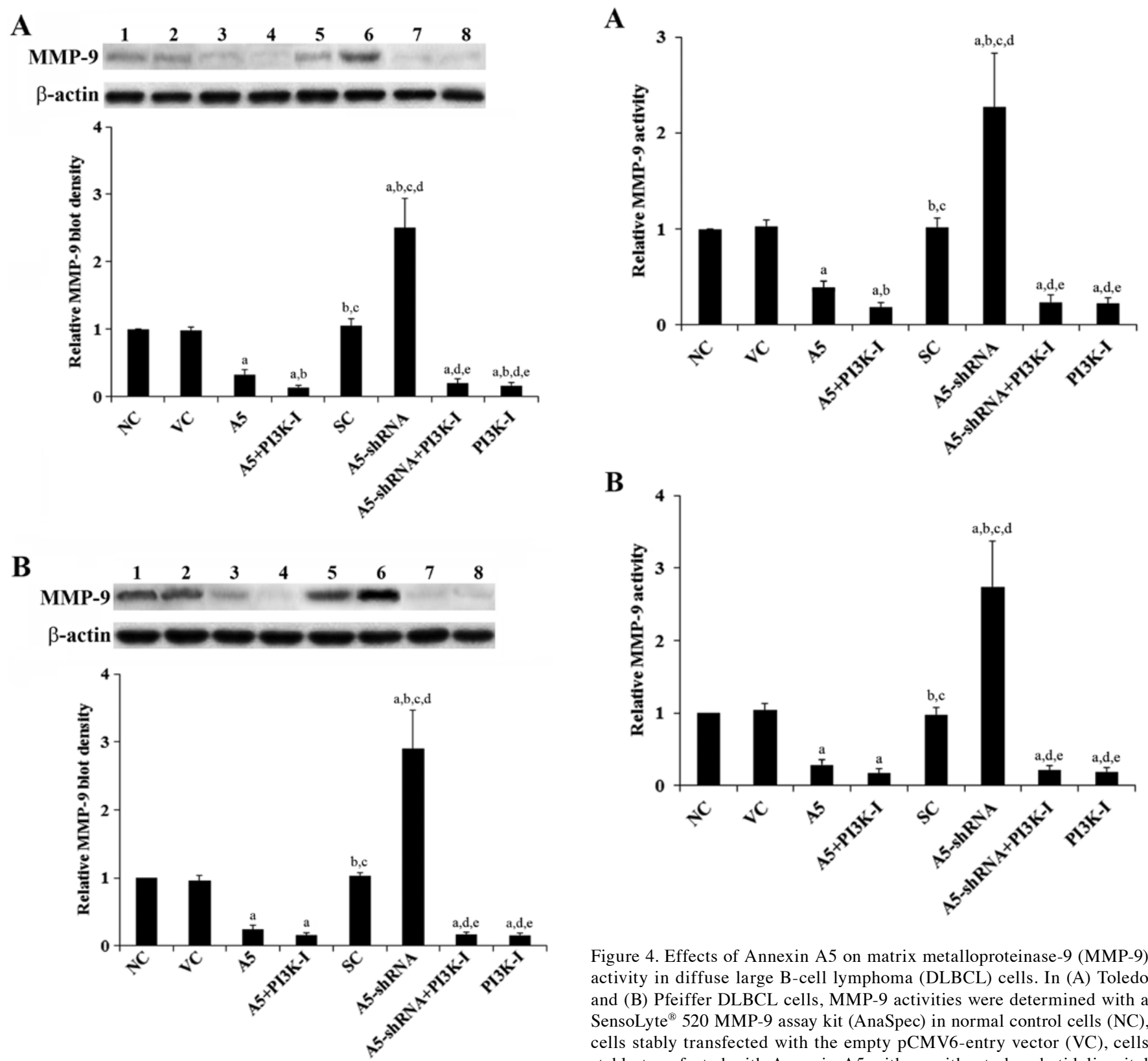

B

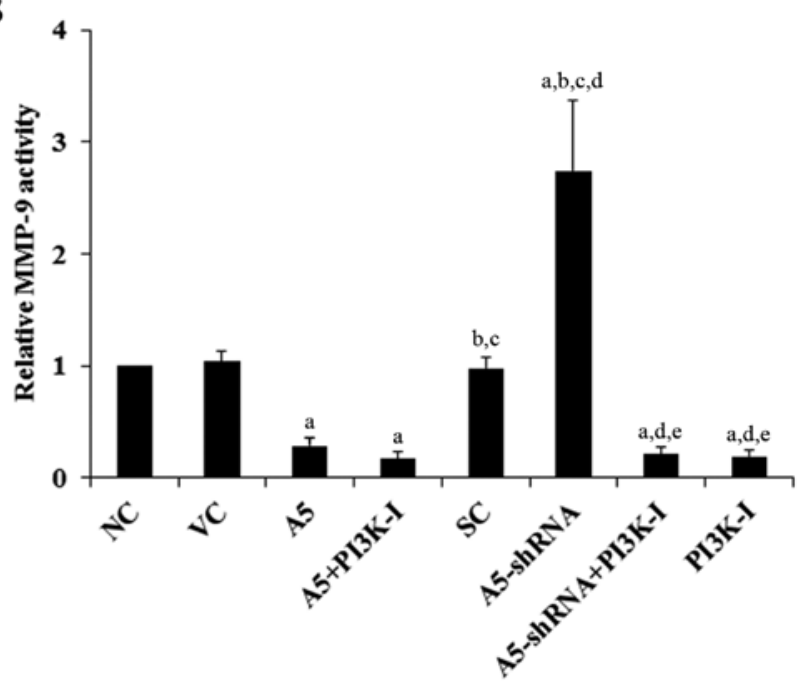

Figure 4. Effects of Annexin A5 on matrix metalloproteinase-9 (MMP-9) activity in diffuse large B-cell lymphoma (DLBCL) cells. In (A) Toledo and (B) Pfeiffer DLBCL cells, MMP-9 activities were determined with a SensoLyte ${ }^{\circledR} 520$ MMP-9 assay kit (AnaSpec) in normal control cells (NC), cells stably transfected with the empty pCMV6-entry vector (VC), cells stably transfected with Annexin A5 with or without phosphatidylinositol 3-kinase (PI3K) inhibitor BKM120 $(50 \mu \mathrm{M})$ treatment (A5; A5+PI3K-I), cells stably transduced with scramble control shRNA (SC), cells stably transduced with Annexin A5-shRNA with or without BKM120 (50 $\mu \mathrm{M})$ treatment (A5-shRNA; A5-shRNA+PI3K-I), and cells treated with BKM120 $(50 \mu \mathrm{M})$ (PI3K-I). The MMP-9 activity was shown as fold changes to that of NC (designated as 1). Each experiment was repeated three times in duplicates. Data values are expressed as mean $\pm \mathrm{SD}$. ${ }^{\mathrm{a}} \mathrm{p}<0.05$ compared with $\mathrm{NC}$ and VC; ${ }^{b} \mathrm{p}<0.05$ compared with A5; ${ }^{\mathrm{p}}<0.05$ compared with A5+PI3K-I; ${ }^{\mathrm{d}} \mathrm{p}<0.05$ compared with $\mathrm{SC} ;{ }^{\mathrm{e}} \mathrm{p}<0.05$ compared with A5-shRNA. BKM120 $(50 \mu \mathrm{M})$ treatment (A5-shRNA, lane 6; A5-shRNA+PI3K-I, lane 7), and cells treated with BKM120 $(50 \mu \mathrm{M})$ (PI3K-I, lane 8$)$ was analyzed with western blot analysis. $\beta$-actin blotting was used as a loading control. Density of the MMP-9 blot was normalized against that of the $\beta$-actin blot to obtain a relative MMP-9 blot density, which was expressed as fold changes to that of NC (designated as 1). Three independent experiments were performed for each western blot analysis. Data values are expressed as mean \pm SD. ${ }^{a} \mathrm{p}<0.05$ compared with NC and VC; ${ }^{b} \mathrm{p}<0.05$ compared with A5; ${ }^{\mathrm{p}} \mathrm{p}<0.05$ compared with A5+PI3K-I; ${ }^{\mathrm{p}} \mathrm{p}<0.05$ compared with SC; ${ }^{\mathrm{e}} \mathrm{p}<0.05$ compared with A5-shRNA.

on DLBCL cell invasion, we performed in vitro cell invasion assays. Compared with the controls, overexpression of Annexin A5 decreased cell invasion by $>60 \%$ in both Toledo and Pfeiffer cells (Fig. 2). On the other hand, knockdown of

Annexin A5 respectively increased cell invasion by 2.1 -fold in Toledo cells and by 2.6-fold in Pfeiffer cells, which was abolished by BKM120 (50 $\mu$ M) (Fig. 2).

MMPs play a critical role in cancer cell invasion (19). Among different MMPs tested, we found that the MMP-9 expression was significantly altered by Annexin A5 in DLBCL cells. As shown in Fig. 3, compared with the controls, overexpression of Annexin A5 decreased MMP-9 expression by $>70 \%$ in both Toledo and Pfeiffer cells. In contrast, knockdown of Annexin A5 respectively increased MMP-9 expression by 

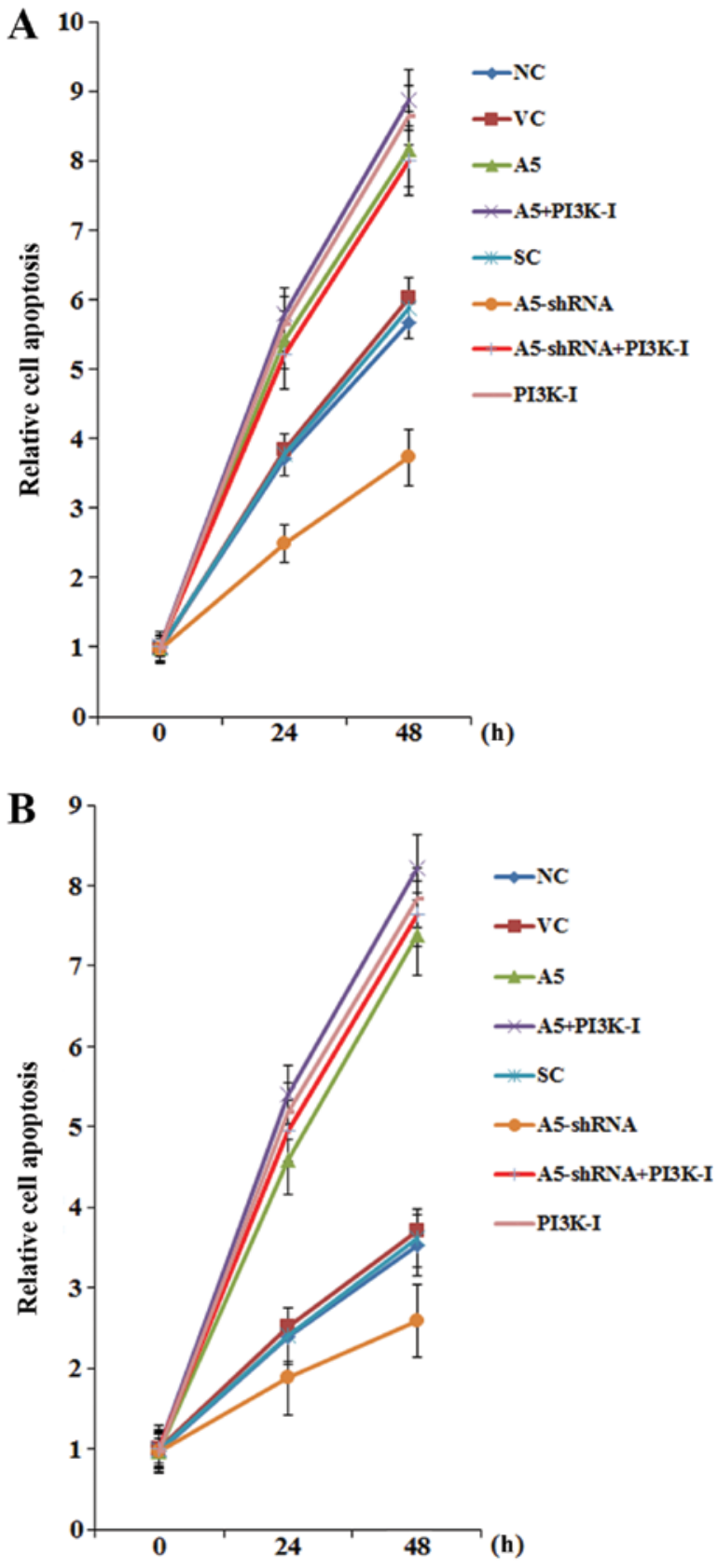

Figure 5. Effects of Annexin A5 on cyclophosphamide, doxorubicin, vincristine and prednisone (CHOP)-induced apoptosis in diffuse large B-cell lymphoma (DLBCL) cells. (A) Toledo and (B) Pfeiffer DLBCL cells were treated with CHOP for 24 and $48 \mathrm{~h}$. The composition of CHOP consisted of cyclophosphoramide, doxorubicin, vincristine and prednisone at the clinical ratio of 80/5.5/0.16/11.1, with the combined CHOP concentration set at $80 \mathrm{ng} / \mathrm{ml}$. Apoptosis was measured with a microplate reader-based TiterTACS in situ apoptosis detection kit (R\&D Systems) in normal control cells (NC), cells stably transfected with the empty pCMV6-entry vector (VC), cells stably transfected with Annexin A5 with or without phosphatidylinositol 3-kinase (PI3K) inhibitor BKM120 $(50 \mu \mathrm{M})$ treatment (A5 A5+PI3K-I), cells stably transduced with scramble control shRNA (SC), cells stably transduced with Annexin A5-shRNA with or without BKM120 $(50 \mu \mathrm{M})$ treatment (A5-shRNA; A5-shRNA+PI3K-I), and cells treated with BKM120 $(50 \mu \mathrm{M})(\mathrm{PI} 3 \mathrm{~K}-\mathrm{I})$. Cell apoptosis was shown as fold changes to that of $\mathrm{NC}$ at $0 \mathrm{~h}$ (designated as 1). Each experiment was repeated three times in triplicates. Data values are expressed as mean \pm SD.

2.5-fold in Toledo cells and by 2.9-fold in Pfeiffer cells, which was abolished by BKM120 $(50 \mu \mathrm{M})($ Fig. 3). Similar data trend was observed with the MMP-9 activity (Fig. 4).
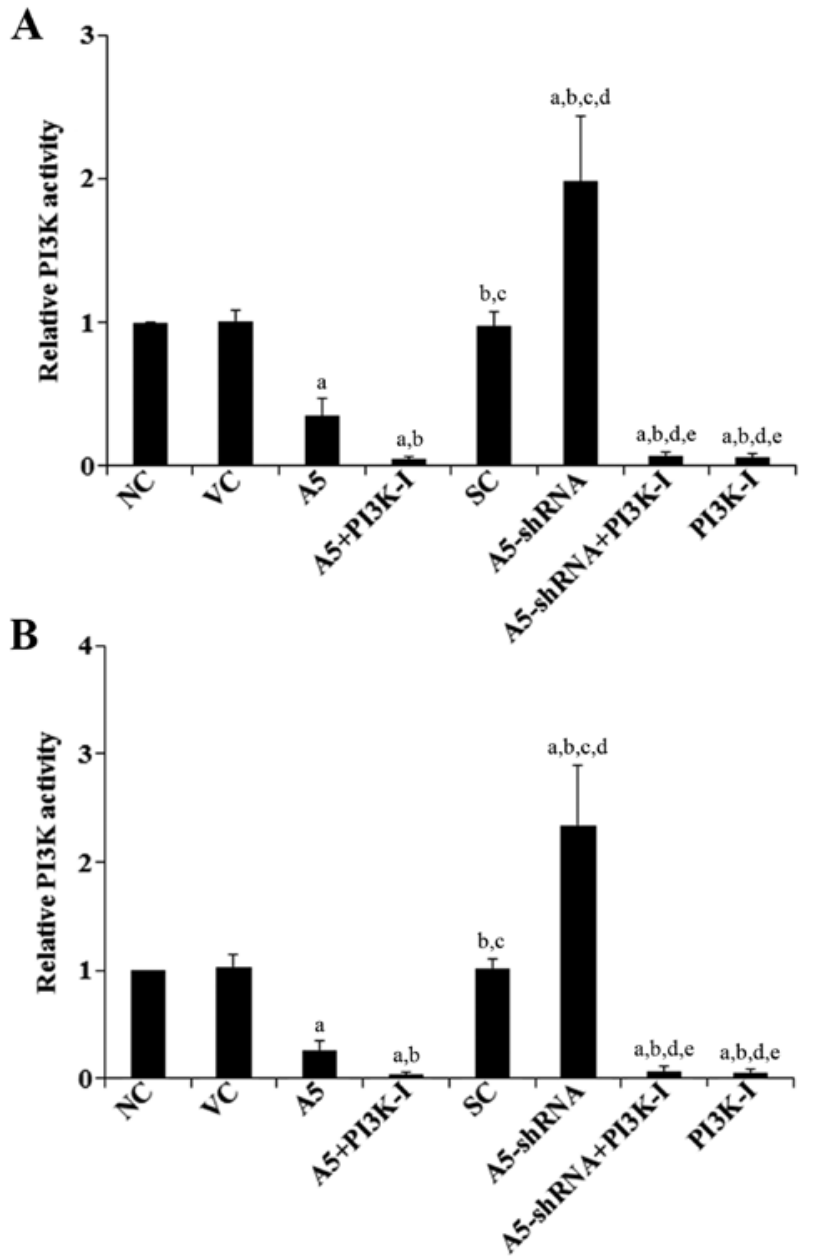

Figure 6. Effects of Annexin A5 on phosphatidylinositol 3-kinase (PI3K) activity in diffuse large B-cell lymphoma (DLBCL) cells. In (A) Toledo and (B) Pfeiffer DLBCL cells, PI3K activities were determined with a PI3K Activity ELISA kit (Echelon Biosciences) in normal control cells (NC), cells stably transfected with the empty pCMV6-entry vector (VC), cells stably transfected with Annexin A5 with or without phosphatidylinositol 3-kinase (PI3K) inhibitor BKM120 $(50 \mu \mathrm{M})$ treatment (A5; A5+PI3K-I), cells stably transduced with scramble control shRNA (SC), cells stably transduced with Annexin A5-shRNA with or without BKM120 $(50 \mu \mathrm{M})$ treatment (A5-shRNA; A5-shRNA+PI3K-I), and cells treated with BKM120 $(50 \mu \mathrm{M})$ (PI3K-I). The PI3K activity was shown as fold changes to that of NC (designated as 1). Each experiment was repeated three times in duplicates. Data values are expressed as mean $\pm \mathrm{SD}$. ${ }^{\mathrm{a}} \mathrm{p}<0.05$ compared with $\mathrm{NC}$ and $\mathrm{VC}$; ${ }^{\mathrm{b}} \mathrm{p}<0.05$ compared with A5; ${ }^{\mathrm{c}} \mathrm{p}<0.05$ compared with A5+PI3K-I; ${ }^{\mathrm{d}} \mathrm{p}<0.05$ compared with SC; ${ }^{\mathrm{e}} \mathrm{p}<0.05$ compared with A5-shRNA.

Effects of Annexin A5 on DLBCL cell chemoresistance to $C H O P$. To explore the effect of Annexin A5 on DLBCL chemoresistance, we examined cell apoptosis in DLBCL cells treated with CHOP in vitro. Overexpression or knockdown of Annexin A5 did not significantly alter cell apoptosis in either Toledo or Pfeiffer cells under normal culture conditions (data not shown). However, overexpression of Annexin A5 significantly increased CHOP-induced DLBCL cell apoptosis compared with the controls (Fig. 5). On the other hand, knockdown of Annexin A5 markedly decreased DLBCL cell apoptosis during CHOP treatment, which was abolished by BKM120 (50 $\mu \mathrm{M})$ (Fig. 5).

Effects of Annexin A5 on PI3K activity and phosphorylation of Akt in DLBCL cells. The above results suggested that 


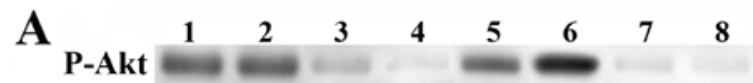

Total Akt
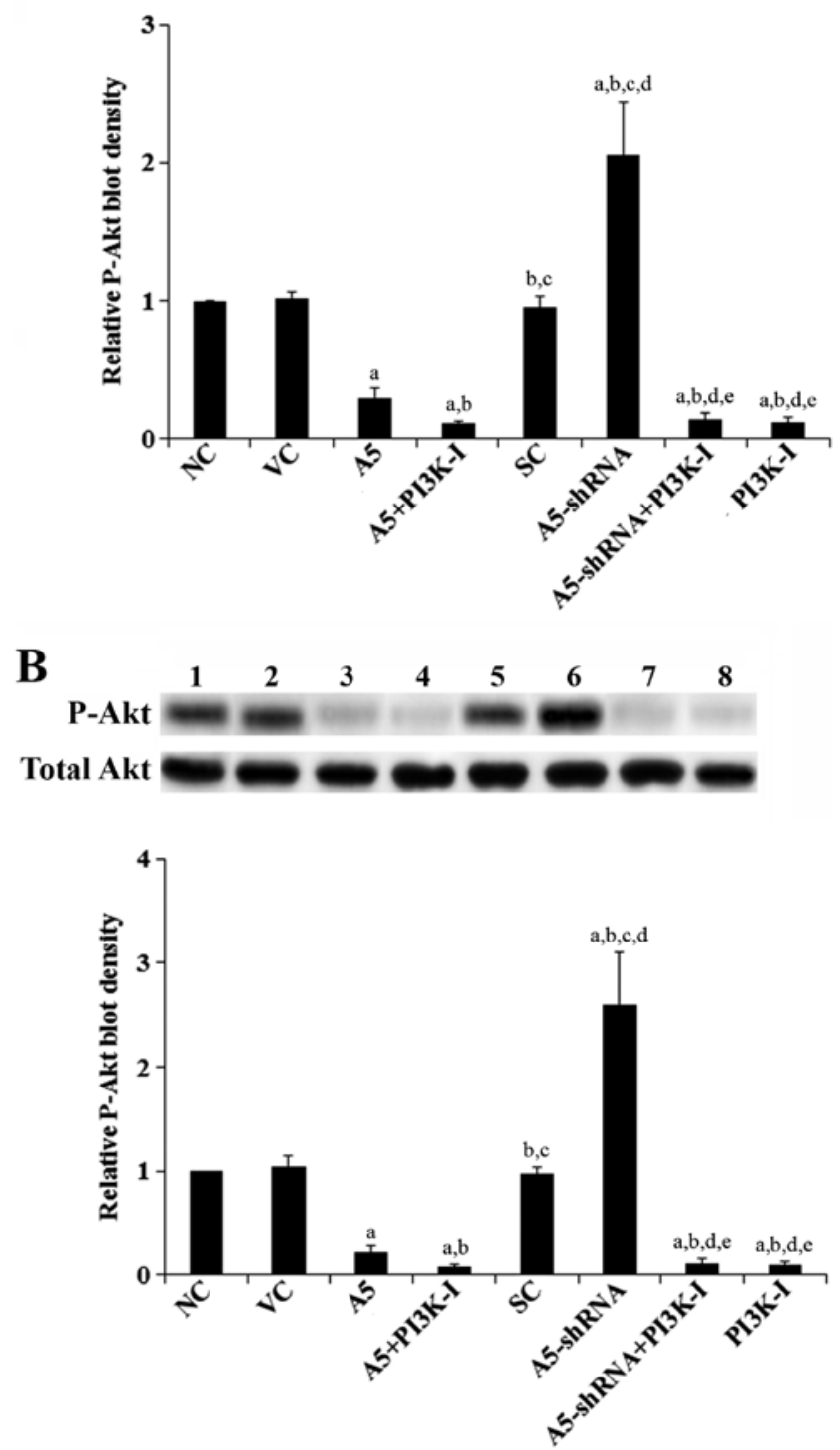

Figure 7. Effects of Annexin A5 on phosphorylated Akt (P-Akt) level in diffuse large B-cell lymphoma (DLBCL) cells. In (A) Toledo and (B) Pfeiffer DLBCL cells, levels of total Akt and P-Akt at serine 473 (ser473) were determined by western blot analyses in normal control cells (NC, lane 1), cells stably transfected with the empty pCMV6-entry vector (VC, lane 2), cells stably transfected with Annexin A5 with or without phosphatidylinositol 3-kinase (PI3K) inhibitor BKM120 $(50 \mu \mathrm{M})$ treatment (A5, lane 3; A5+PI3K-I, lane 4), cells stably transduced with scramble control shRNA (SC, lane 5), cells stably transduced with Annexin A5-shRNA with or without BKM120 $(50 \mu \mathrm{M})$ treatment (A5-shRNA, lane 6; A5-shRNA+PI3K-I, lane 7), and cells treated with BKM120 (50 $\mu \mathrm{M})$ (PI3K-I, lane 8). The total Akt level was not significantly altered by Annexin A5 in both Toledo and Pfeiffer cells. Density of the P-Akt (ser473) blot was normalized against that of total Akt to obtain a relative P-Akt blot density, which was expressed as fold changes to that of NC (designated as 1). Three independent experiments were performed for each western blot analysis. Data values are expressed as mean \pm SD. ${ }^{a} \mathrm{p}<0.05$ compared with $\mathrm{NC}$ and $\mathrm{VC} ;{ }^{\mathrm{b}} \mathrm{p}<0.05$ compared with A5; ${ }^{c} \mathrm{p}<0.05$ compared with A5+PI3K-I; ${ }^{d} \mathrm{p}<0.05$ compared with SC; ${ }^{e} \mathrm{p}<0.05$ compared with A5-shRNA.

Annexin A5 inhibited DLBCL cell chemoresistance to CHOP through a PI3K-dependent mechanism. Indeed, the PI3K/ Akt pathway is reportedly important for cancer cell chemo- resistance (20-23). Thus, we next examined the effects of Annexin A5 on the PI3K activity and phosphorylation of Akt in DLBCL cells. As shown in Fig. 6, compared with the controls, overexpression of Annexin A5 decreased the PI3K activity by $\sim 65 \%$ in Toledo cells and by $75 \%$ in Pfeiffer cells, respectively. In contrast, knockdown of Annexin A5 respectively increased the PI3K activity by 2 -fold in Toledo cells and by 2.3-fold in Pfeiffer cells, which was abolished by BKM120 $(50 \mu \mathrm{M})$ (Fig. 6). Similar data trend was observed with phosphorylation at serine 473 (ser473) of Akt (Fig. 7), which is required for full activation of Akt by PI3K.

\section{Discussion}

CHOP is widely used in China to treat DLBCL. Although patient outcomes have significantly improved to a $>40 \%$ cure rate by $\mathrm{CHOP}$ chemotherapy, resistance to the $\mathrm{CHOP}$ regimen continues to pose a challenge in managing or curing DLBCL (3). Understanding the molecular basis for development of multi-drug chemoresistance in DLBCL may serve as a basis for identification of novel therapeutic targets and biomarkers involved in DLBCL resistance to CHOP. Previous studies have suggested that Annexin A5, a calcium-dependent phospholipid-binding protein, while promoting the malignancy and chemoresistance in certain types of cancer, is negatively correlated with those in other cancers, including DLBCL (9-12). In the present study, we demonstrated an important inhibitory role for Annexin A5 in DLBCL cell invasion and chemoresistance to CHOP.

DLBCL is a heterogeneous group of tumors with a high variance of genetic abnormalities, clinical features, response to treatment and prognosis (2). Thus, we used two DLBCL cell lines with considerable background differences (respectively established from peripheral blood leukocytes and metastatic site of DLBCL patients) as cell models in this study to demonstrate a generalizable role of Annexin A5 in DLBCL cell invasion and chemoresistance to CHOP. In both cell lines, while overexpression of Annexin A5 significantly decreased cell invasion and cell survival against CHOP-induced apoptosis, knockdown of Annexin A5 markedly increased cell invasion and CHOP-induced apoptosis, which was abolished by a selective PI3K inhibitor. The results suggest that Annexin A5 inhibited DLBCL cell invasion and chemoresistance to CHOP through a PI3K-depedent mechanism. This was corroborated by the results that knockdown of Annexin A5 increased the PI3K activity and Akt phosphorylation in DLBCL cells. The findings are also in line with previous studies that the PI3K/Akt pathway plays a critical role in cancer cell invasiveness and chemoresistance (20-23).

MMPs play a critical role in cancer cell invasion (19). Among different MMPs tested, we found that the MMP-9 expression/activity was significantly altered by Annexin A5, which showed similar data trend as that in DLBCL cell invasion. Previous studies have shown that PI3K signaling can stimulate MMP-9 expression $(24,25)$. In our study, overexpression of Annexin A5 in DLBCL cells significantly decreased the PI3K activity, MMP-9 expression/activity, and cell invasion. On the other hand, knockdown of Annexin A5 markedly increased the PI3K activity as well as MMP-9 expression/activity and cell invasion, which was abolished 
by a selective PI3K inhibitor. The findings suggest that Annexin A5 could inhibit DLBCL cell invasion by downregulating the MMP-9 expression/activity through inhibiting PI3K activity/signaling.

It has been reported that Annexin A5 dysexpression may lead to deregulated activation of PKC and abnormality of cellular signal transduction, which may be involved in carcinogenesis (9). In our study, however, altered expression of Annexin A5 in DLBCL cells only showed major regulatory effects on PI3K activity/signaling, which subsequently led to significant changes in cell invasion and chemoresistance to CHOP. Future studies are required to examine the mechanism by which Annexin A5 inhibits PI3K activity/signaling in DLBCL cells.

Annexin A5 is negatively correlated with the malignancy and chemoresistance in some cancers, while it enhances those in other cancers (9-12). Our study demonstrated an inhibitory effect of Annexin A5 on DLBCL chemoresistance, while previous studies have shown that Annexin A5 enhances chemoresistance in gastric cancer and nasopharyngeal carcinoma $(10,11)$. Thus, Annexin A5 likely plays a dual role in cancer cell malignancy and chemoresistance, depending on tissue specificity. The functional role of Annexin A5 in DLBCL invasiveness and chemoresistance will be verified in more DLBCL cell lines and in animal models in our future studies.

Twelve Annexins common to vertebrates are known as Annexins A1-A11 and A13 (1,2). Annexin A1, A2, A4 and A5 play important roles in breast, pancreatic and laryngeal carcinoma, alone and/or synergistically, which has made them potential therapeutic targets for malignant tumors (26). Based on our findings in the present study, it is noteworthy to investigate whether other Annexin family members play a role, alone or in combination with Annexin A5, in DLBCL cell invasion and chemoresistance.

In conclusion, our study provides the first in vitro evidence that Annexin A5 inhibits DLBCL cell invasion, MMP-9 expression/activity, and chemoresistance to CHOP through a PI3K-dependent mechanism. It provides new insight not only into the biological function of Annexin A5, but also into the molecular mechanisms underlying DLBCL progression and chemoresistance.

\section{References}

1. Campo E, Swerdlow SH, Harris NL, Pileri S, Stein H and Jaffe ES: WHO classification of tumours of hematopoietic and lymphoid tissues. Blood 117: 5019-5032, 2011.

2. Lossos IS and Morgensztern D: Prognostic biomarkers in diffuse large B-cell lymphoma. J Clin Oncol 24: 995-1007, 2006.

3. Maxwell SA, Li Z, Jaya D, Ballard S, Ferrell J and Fu H: 14-33zeta mediates resistance of diffuse large B cell lymphoma to an anthracycline-based chemotherapeutic regimen. J Biol Chem 284: 22379-22389, 2009.

4. Shipp MA, Ross KN, Tamayo P, et al: Diffuse large B-cell lymphoma outcome prediction by gene-expression profiling and supervised machine learning. Nat Med 8: 68-74, 2002.

5. Moss SE and Morgan RO: The annexins. Genome Biol 5: 219, 2004.
6. Laohavisit A and Davies JM: Multifunctional annexins. Plant Sci 177: 532-539, 2009.

7. Mussunoor S and Murray GI: The role of annexins in tumour development and progression. J Pathol 216: 131-140, 2008.

8. Nakao $H$, Watanabe $M$ and Maki $M$ : A new function of calphobind I (annexin V). Eur J Biochem 223: 901-908, 1994.

9. Peng B, Guo C, Guan H, Liu S and Sun MZ: Annexin A5 as a potential marker in tumors. Clin Chim Acta 427: 42-48, 2014.

10. Wu X, Tang Y, Huang W, et al: Identification of proteins interacting with multidrug resistance protein in gastric cancer. World J Gastroenterol 19: 3568-3573, 2011.

11. Tang S, Huang W, Zhong M, et al: Identification keratin 1 as a cDDP-resistant protein in nasopharyngeal carcinoma cell lines. J Proteomics 75: 2352-2360, 2012.

12. Liu Y, Zeng L, Zhang S, Zeng S, Huang J, Tang Y and Zhong M: Identification of differentially expressed proteins in chemotherapy-sensitive and chemotherapy-resistant diffuse large B cell lymphoma by proteomic methods. Med Oncol 30: 528, 2013.

13. Wang B, Feng P, Xiao Z and Ren EC: LIM and SH3 protein 1 (Lasp1) is a novel p53 transcriptional target involved in hepatocellular carcinoma. J Hepatol 50: 528-537, 2009.

14. Feng Y, Hu J, Ma J, et al: RNAi-mediated silencing of VEGF-C inhibits non-small cell lung cancer progression by simultaneously down-regulating the CXCR4, CCR7, VEGFR-2 and VEGFR-3dependent axes-induced ERK, p38 and AKT signalling pathways. Eur J Cancer 47: 2353-2363, 2011.

15. Jo YK, Park SJ, Shin JH, Kim Y, Hwang JJ, Cho DH and Kim JC: ARP101, a selective MMP-2 inhibitor, induces autophagy-associated cell death in cancer cells. Biochem Biophys Res Commun 404: 1039-1043, 2011.

16. Qazi H, Shi ZD and Tarbell JM: Fluid shear stress regulates the invasive potential of glioma cells via modulation of migratory activity and matrix metalloproteinase expression. PLoS One 6: e20348, 2011.

17. Cao CM,Zhang Y, Weisleder N, et al: MG53 constitutes a primary determinant of cardiac ischemic preconditioning. Circulation 121: 2565-2574, 2010.

18. Fos C, Salles A, Lang V, et al: ICOS ligation recruits the p50alpha PI3K regulatory subunit to the immunological synapse. J Immunol 181: 1969-1977, 2008.

19. Li Y, Liao Q, Li K, Zhong D, Weng X and Mi M: Knockdown of endothelin A receptor expression inhibits osteosarcoma pulmonary metastasis in an orthotopic xenograft mouse model. Mol Med Rep 5: 1391-1395, 2012.

20. Li B, Yang Y, Jiang S, Ni B, Chen K and Jiang L: Adenovirusmediated overexpression of BMP-9 inhibits human osteosarcoma cell growth and migration through downregulation of the PI3K/ AKT pathway. Int J Oncol 41: 1809-1819, 2012.

21. Liu ZL, Mao JH, Peng AF, Yin QS, Zhou Y, Long XH and Huang SH: Inhibition of fatty acid synthase suppresses osteosarcoma cell invasion and migration via downregulation of the PI3K/Akt signaling pathway in vitro. Mol Med Rep 7: 608-612, 2013.

22. Zhao G, Cai C, Yang T, et al: MicroRNA-221 induces cell survival and cisplatin resistance through PI3K/Akt pathway in human osteosarcoma. PLoS One 8: e53906, 2013.

23. Wang TF, Wang $\mathrm{H}$, Peng AF, et al: Inhibition of fatty acid synthase suppresses U-2 OS cell invasion and migration via downregulating the activity of HER2/PI3K/AKT signaling pathway in vitro. Biochem Biophys Res Commun 440: 229-234, 2013.

24. Qin J, Tang J, Jiao L, et al: A diterpenoid compound, excisanin A, inhibits the invasive behavior of breast cancer cells by modulating the integrin $\beta 1 / \mathrm{FAK} / \mathrm{PI} 3 \mathrm{~K} / \mathrm{AKT} / \beta$-catenin signaling. Life Sci 93: 655-663, 2013.

25. Lin G, Sun L, Wang R, Guo Y and Xie C: Overexpression of muscarinic receptor 3 promotes metastasis and predicts poor prognosis in non-small-cell lung cancer. J Thorac Oncol 9: 170-178, 2014.

26. Deng S, Wang J, Hou L, et al: Annexin A1, A2, A4 and A5 play important roles in breast cancer, pancreatic cancer and laryngeal carcinoma, alone and/or synergistically. Oncol Lett 5: 107-112, 2013. 